\title{
Foreword
}

\section{Atrial Fibrillation Risk Factors}

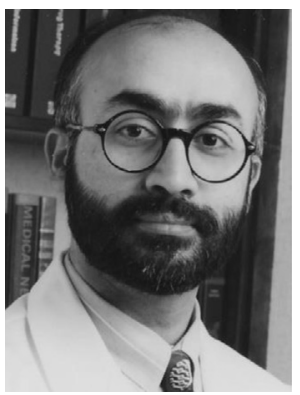

Ranjan K. Thakur, MD, MPH, MBA, FHRS

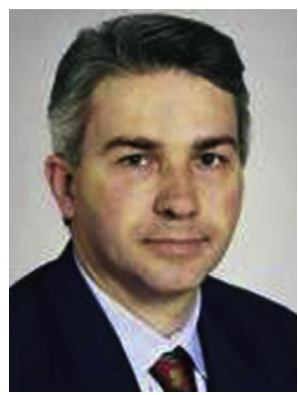

Andrea Natale, MD, FACC, FHRS

Consulting Editors

Atrial fibrillation (AF) is the most common sustained cardiac arrhythmia seen in clinical practice. When we started our clinical careers about 25 years ago, electrophysiologists were usually consulted for other arrhythmia issues, such as syncope, ventricular tachycardia, implantable cardioverter defibrillator implantation, supraventricular tachycardia management, and AF management was not a very prominent part of our clinical practice. Then came Haissaguerre's seminal paper showing that AF can be triggered by rapid electrical firing from the pulmonary veins. This incited worldwide interest in trying to understand and successfully ablate AF. Concerted effort by basic scientists, clinicians, and industry has brought us to the point where the majority of an electrophysiologist's clinical effort is exerted on managing AF.

Our understanding has grown tremendously. Patient selection is more informed. Safety of the ablation procedure has improved. We are better informed about progressively sequencing the right lesion sets in a patient. But unfortunately, the clinical results of catheter ablation have remained more or less the same.

We need to develop other approaches to tackling $A F$ to improve outcomes. A deeper understanding of $A F$ risk factors, risk factor modification, and lifestyle modification may be beneficial. Research has demonstrated that multiple risk factors predispose to AF. Among these are family history (genes), hypertension, heart failure, diabetes, obesity, sleep apnea, smoking, pulmonary disease, cardiomyopathies, endocrine abnormalities, valvular heart disease and coronary artery disease, alcohol consumption, and air pollution. Although these predisposing factors are well discussed individually, an amalgamated comprehensive review by experts in the field was absent.

We congratulate the editors, Drs Shenasa, Nattel, and Sanders, for a timely issue on this important approach to deepen our understanding of $\mathrm{AF}$, and it is hoped, improving future therapeutic outcomes. This issue will be of interest to clinicians and researchers. We hope that the readership will enjoy reading the individual articles.

Ranjan K. Thakur, MD, MPH, MBA, FHRS Sparrow Thoracic and Cardiovascular Institute Michigan State University 1440 East Michigan Avenue; Suite 400 Lansing, MI 48912, USA

Andrea Natale, MD, FACC, FHRS Texas Cardiac Arrhythmia Institute

Center for Atrial Fibrillation at

St. David's Medical Center 1015 East 32nd Street, Suite 516

Austin, TX 78705, USA

E-mail addresses:

epthakur@gmail.com (R.K. Thakur) andrea.natale@stdavids.com (A. Natale) 